\title{
Time evolution of the fluid flow at the top of the core. Geomagnetic jerks
}

\author{
Minh Le Huy ${ }^{1,2}$, Mioara Mandea ${ }^{1}$, Jean-Louis Le Mouël ${ }^{1}$, and Alexandra Pais ${ }^{1}$ \\ ${ }^{1}$ Institut de Physique du Globe de Paris, B.P. 89, 4 Place Jussieu, 75252 Paris cedex 5, France \\ ${ }^{2}$ Hanoi Institute of Geophysics, Nghia do - Tu Liem - Hanoi - Vietnam
}

(Received August 30, 1999; Revised November 15, 1999; Accepted November 15, 1999)

\begin{abstract}
The knowledge of the geomagnetic field and its secular variation allows us to compute the fluid flow at the core surface. The poloidal and toroidal components of the fluid flow at the core-mantle boundary (CMB) have been calculated every year from the Bloxham and Jackson model (1992) and plotted at 50 year intervals over the last three centuries. The flow patterns conserve some broad features over this whole time-span. The time constant of the degree 1 component of the motion is larger than the time constant of the rest of the flow. The average motion over 300 years appears to be in large part symmetrical with respect to the equator. This average flow can be represented by the sum of a few geostrophic vectors. The acceleration fields corresponding to the well documented jerks of 1969, 1979, 1992 have also been computed. The geometry of these acceleration fields is the same, within a change of sign, for the three events. Moreover, this geometry has close connections with the geometry of the flow itself. The spatial and temporal variations of the flow field can be simply described, in a first approximation; it is possible to give an analytical schematic representation of the flow field during the last three decades. Some characteristics of the decadal length-of-day variations follow if the coupling torque between core and mantle is topographic.
\end{abstract}

\section{Introduction}

The last 20 years (since MAGSAT) have seen spectacular advances in our ability to generate realistic maps of the Earth's magnetic field outside the planet and at the interface between the fluid core and the overlying solid mantle, using data collected at or above the Earth's surface. Studies of the field over the last 300 years have revealed some remarkably stable features in the morphology of the field, and it has been discussed whether these features might even have been stable over palaeomagnetic time-scales. Temporal variations are nevertheless conspicuous in data from the last few hundred years, and these variations have implications for the dynamics of the fluid flow at the core-mantle boundary and the coupling mechanism between the core and mantle.

In this paper we will first rely on maps and graphs to single out simple basic features of the behavior of the geomagnetic field in time and space. We will then infer a simplified analytic expression of this time-space behavior and use it to suggest an interpretation of decadal length-of-day variations.

\section{Evaluation of the Main Field Variation}

Until recent decades, maps and models of the geomagnetic field were usually established for navigation, rather than for understanding the physical process that generates the Earth's main magnetic field. First restricted to either long time periods sparsely sampled (Fritsche, 1903; Braginsky, 1972; Benkova et al., 1974 (BKC hereafter); Barraclough, 1974) or to short periods described with some temporal details (Malin,

Copy right $(\mathrm{C}$ The Society of Geomagnetism and Earth, Planetary and Space Sciences (SGEPSS); The Seismological Society of Japan; The Volcanological Society of Japan; The Geodetic Society of Japan; The Japanese Society for Planetary Sciences.
1969; Malin and Clark, 1974), models gained more and more accuracy, involved longer time-spans and were produced in a more and more homogeneous way over the period considered.

The Bloxham and Jackson model (1992) (BJ hereafter) is unquestionably the most complete and homogeneous historical description of the main magnetic field presently available. The spatial dependency of the field is represented by a spherical harmonic expansion, and the time dependency by a cubic $\mathrm{B}$-spline basis. The expansion in spherical harmonics of the BJ models is limited to order and degree 14. For the temporal cubic B-spline basis the knot points have been chosen every 2.5 years. The model consists of two parts (before and after 1840, a limit imposed by the absence of field intensity measurements before this date). The resulting model covers three centuries (1690-1990).

The corresponding maps of the vertical component of the field $B_{r}(t)$ at the CMB from 1690 to 1990 are well-known, in the form of a sequence of snapshots at 50 years intervals (see again Bloxham and Jackson, 1992). In the following we only recall some prominent features of the field at the core mantle boundary (CMB). A remarkable standing feature consists of two pairs of intense flux patches, one located under Artic Canada and south-east of Tierra del Fuego and the other under Siberia and south-east of Australia. They have remained largely the same for 300 years (Bloxham and Gubbins, 1985). These two pairs of enhanced positive-negative flux patches have been seen as the signature of convective rolls (Gubbins and Bloxham, 1987). Gubbins and Kelly (1993) and Kelly and Gubbins (1997) argue that the two pairs of patches are still present, although subdued, on maps derived from palaeo- 


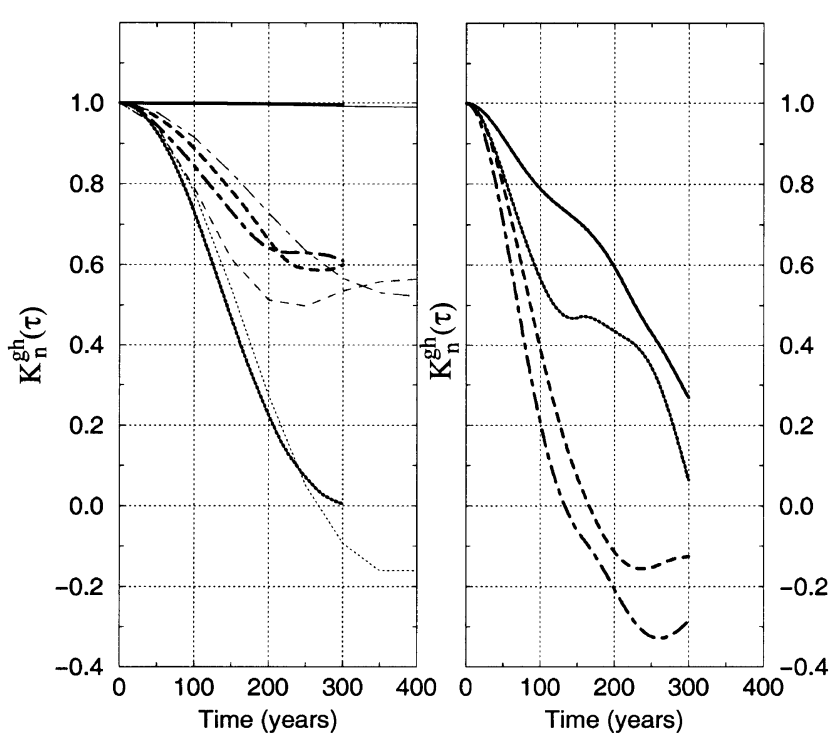

Fig. 1. Auto-correlation coefficients for different degrees of the geomagnetic field models considered in this study (thin lines - BCK model, thick lines - BJ model). Left: $n=1$ (solid line), $n=2$ (dotted line), $n=3$ (dashed line) and $n=4$ (dot-dashed line). Right: $n=5$ (solid line), $n=6$ (dotted line), $n=7$ (dashed line) and $n=8$ (dot-dashed line).

magnetic data averaged over one million years. But Carlut and Courtillot $(1995,1998)$ claimed that no significant nonzonal term exists in the models derived from palaeomagnetic data. Hulot and Le Mouël (1994) concluded from a study of BJ model that, while the characteristic time constant of the non-dipole terms of the field model is not larger than some 200 years, the probability of features remaining stationary for a few hundreds years is not negligible.

The Hulot and Le Mouël (1994) analysis considered a three centuries time-span constrained by the BJ models. Using the BKC models, one can go one century further in the past. Let us recall the Hulot and Le Mouël analysis. A quantitative estimate of the stability of the different harmonics $(n)$ can be obtained by computing the correlation function:

$$
\begin{aligned}
& K_{n^{g h}(\tau)} \\
& =\frac{1}{P(\tau)} \sum_{p=0}^{P(\tau)} \frac{\sum_{m=0}^{n}\left[g_{n}^{m}(p \delta t) g_{n}^{m}(p \delta t+\tau)\right.}{\left[\left(\sum_{m=0}^{n}\left(g_{n}^{m}(p \delta t)\right)^{2}+\left(h_{n}^{m}(p \delta t)\right)^{2}\right)\right.} \\
& \frac{\left.+h_{n}^{m}(p \delta t) h_{n}^{m}(p \delta t+\tau)\right]}{\left.\left(\sum_{m=0}^{n}\left(g_{n}^{m}(p \delta t+\tau)\right)^{2}+\left(h_{n}^{m}(p \delta t+\tau)\right)^{2}\right)\right]^{1 / 2}}
\end{aligned}
$$

where $P(\tau)=(T-\tau) / \delta t$ is the number of time steps $\delta t$ used to calculate the current correlation function for a given delay $\tau ; T$ is the considered time interval (i.e., $300 \mathrm{yr}$ for the BJ models and $400 \mathrm{yr}$ for the BCK models) and $\delta t$ is the time-step of the geomagnetic models ( $\delta t=1$ year here); $g_{n}^{m}, h_{n}^{m}$ are the Gauss coefficients.

Figure 1 illustrates the results. $K_{1}^{g h}(\tau)$ keeps close to 1 for both models, varying by less than $1 \%$ over four centuries. The decreasing trend for degrees $n=2$ and $n>5$ is con- firmed. Also, the tendency of $K_{3}^{g h}(\tau)$ and $K_{4}^{g h}(\tau)$ to remain stable and larger than 0.5 after 200 years is clear. The probability that such a behavior is compatible with the statistical model of Hulot and Le Mouël is then weaker than considered by the authors when using a shorter 300 years period. The stability of the flux patches is to be related with this longer correlation time of the degrees 3 and 4 harmonics.

Another outstanding feature of the secular variation over the last three centuries is the regular westward drift, at a rate of about $0.28^{\circ}$ year, of a negative spot on the map of the time derivative (secular variation), $\dot{B}_{r}(t)$, of the vertical component of the field centered on the equator from 1700 (see the BJ models). This does not mean a global westward drift of the secular variation field; in fact, a westward motion of the fluid at the CMB concentrated in the vicinity of the equator and limited to the western hemisphere was pointed out some time ago (e.g., Gire et al., 1986; Bloxham and Gubbins, 1985).

In the following we will use the vertical component of the magnetic field $B_{r}$ at the CMB, as well as of its secular variation $\dot{B}_{r}(t)$, both provided by the BJ models, from 1690 to 1990 . For this time-span we will compute and analyse the evolution of the flow at the top of the core.

\section{Computation of the Flow at the CMB}

We first present the evolution of the flow at the CMB on the long term, from 1690 to the present, then for the three last decades of the present century, with more detail.

\subsection{Computation of the flow}

We compute the large-scale flow using the frozen-flux and tangentially geostrophic hypothesis (Roberts and Scott, 1965; Le Mouël, 1984; Gire et al., 1984; Gire and Le Mouël, 1990), which has been supported by recent computations performed locally at the surface of the CMB (Chulliat and Hulot, 1999):

$$
\begin{gathered}
\frac{\partial B_{r}}{\partial t}=-\vec{\nabla}_{H} \cdot\left(\vec{u} B_{r}\right) \\
\vec{u}=\frac{1}{2 \rho \Omega_{0} \cos \theta} \vec{n} \wedge \vec{\nabla} p \\
\vec{\nabla}_{H} \cdot(\vec{u} \cos \theta)=0
\end{gathered}
$$

where $\vec{u}$ is the horizontal velocity field at the surface of the core (of the main stream), $\vec{\nabla}_{H}$ the gradient operator reduced to horizontal coordinates, $\theta$ the co-latitude, $\rho$ is the core density, $\Omega_{0}$ is the Earth's mean rotation, $\vec{n}$ the unit outward radial vector and $\vec{\nabla} p$ the pressure gradient.

The velocity field at the top of the core can be represented as the sum of two terms:

$$
\vec{u}=c \vec{\nabla}_{H} S-c \vec{n} \wedge \vec{\nabla}_{H} T
$$

where $c$ is the core radius. Expanding the poloidal $S$ and toroidal $T$ scalar functions in surface harmonics, $\vec{u}$ can be written in the form:

$$
\begin{aligned}
\vec{u}= & c\left[\sum _ { n = 1 } ^ { \infty } \sum _ { m = 0 } ^ { n } \left(s_{n}^{m c} \vec{S}_{n}^{m c}\right.\right. \\
& \left.\left.+s_{n}^{m s} \vec{S}_{n}^{m s}+t_{n}^{m c} \vec{T}_{n}^{m c}+t_{n}^{m s} \vec{T}_{n}^{m s}\right)\right]
\end{aligned}
$$


where $\vec{S}_{n}^{m(c, s)}$ and $\vec{T}_{n}^{m(c, s)}$ are elementary poloidal and toroidal vectors (e.g., Backus, 1986):

$$
\begin{array}{r}
\vec{S}_{n}^{m(c, s)}=c \vec{\nabla}_{H} Y_{n}^{m(c, s)} \\
\vec{T}_{n}^{m(c, s)}=-c \vec{n} \wedge \vec{\nabla}_{H} Y_{n}^{m(c, s)}
\end{array}
$$

with

$$
Y_{n}^{m(c, s)}=P_{n}^{m}(\cos \theta)(\cos m \phi, \sin m \phi)
$$

where $P_{n}^{m}$ are Schmidt normalised associated Legendre functions. The $s_{n}^{m(c, s)}, t_{n}^{m(c, s)}$ are the poloidal and toroidal coef-


imation, $\vec{u}$ can also be expanded on a basis of elementary geostrophic vectors (Le Mouël et al., 1985; Backus and Le Mouël, 1986):

$$
\vec{u}=c \sum_{n=1}^{\infty}\left[t_{n}^{0} \vec{T}_{n}^{0}+\sum_{m=1}^{n}\left(s_{n}^{m c} \vec{V}_{n}^{m c}+s_{n}^{m s} \vec{V}_{n}^{m s}\right)\right]
$$

where $\vec{T}_{n}^{0}, \vec{V}_{n}^{m(c, s)}$ are the elementary geostrophic vectors. $\vec{u}(t)$ has been computed from $B_{r}(t)$ and $\dot{B}_{r}(t)$, applying the
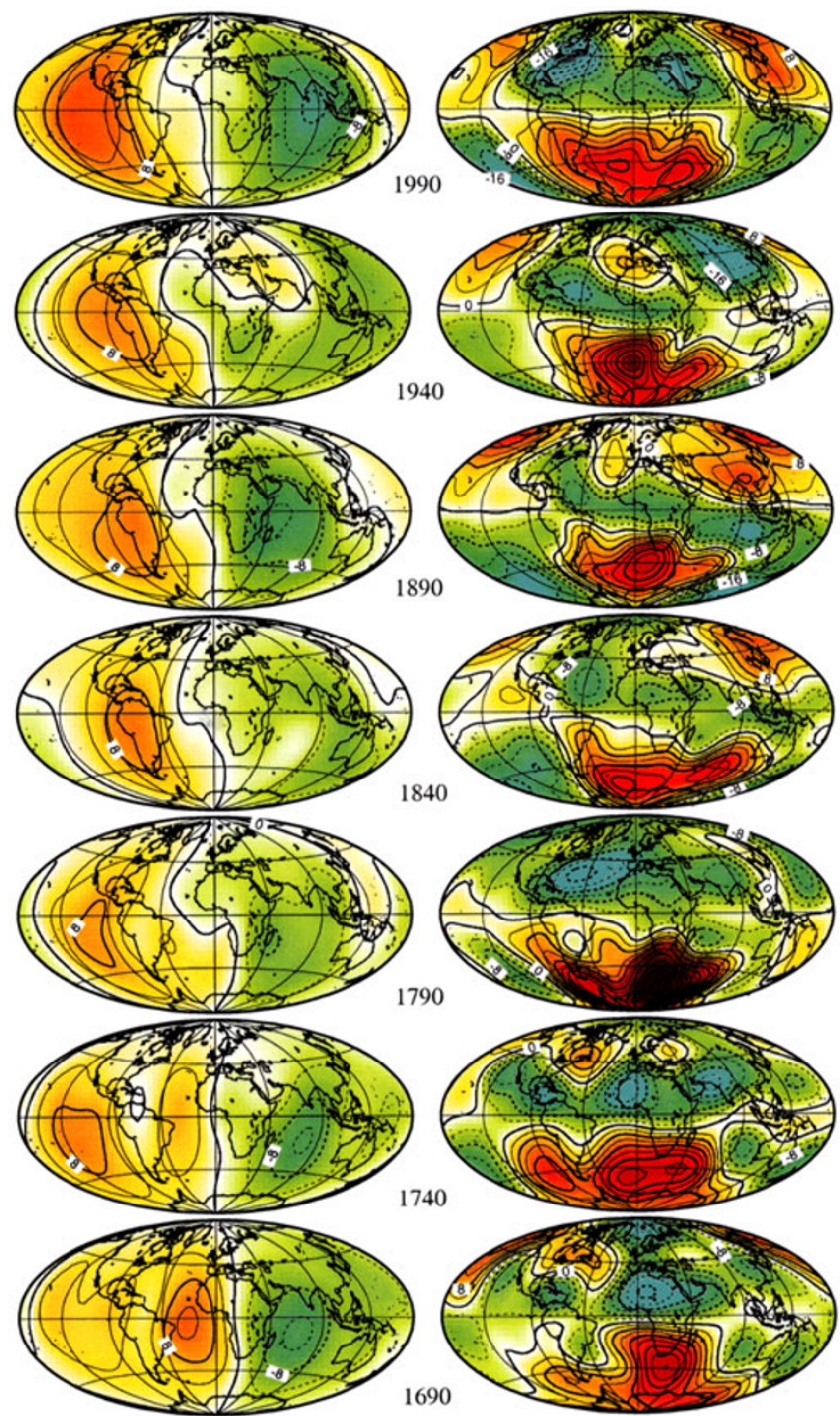

Fig. 2. Poloidal (left) and toroidal (right) scalars, $S(t)$ and $T(t)$, every 50 years. Contour interval: $4 \cdot 10^{-4} \mathrm{rad}_{\mathrm{yr}} \mathrm{r}^{-1}$. inversion method proposed by Gire and Le Mouël (1990) and discussed by Hulot et al. (1992), with an appropriate choice of parameters. The expansions (6) and (10) are truncated at degree 13.

Figure 2 shows the corresponding maps of the poloidal and toroidal scalars $S(t)$ and $T(t)$ every 50 years. In the following discussion, the zonal toroidal part of the flow is not retained because from our point of view it is partly a secondary flow accelerated by the pressure torque exerted by the primary non-zonal flow (Jault and Le Mouël, 1990). A first simple observation can be readily made: the general organization of the flow tends to conserve some gross features over the whole considered time-span (the maps suffer of course from large inaccuracies). Let us examine this in more detail. Figure 3 shows the degree 1 component of the poloidal scalar $\left(a \sin \theta \cos \left(\phi-\phi_{0}\right)\right.$, where $a$ is the amplitude of the component of the first degree); there is no zonal poloidal component in a geostrophic flow) and the degree 2 component of the toroidal scalar (containing $Y_{2}^{1}$ and $Y_{2}^{2}$
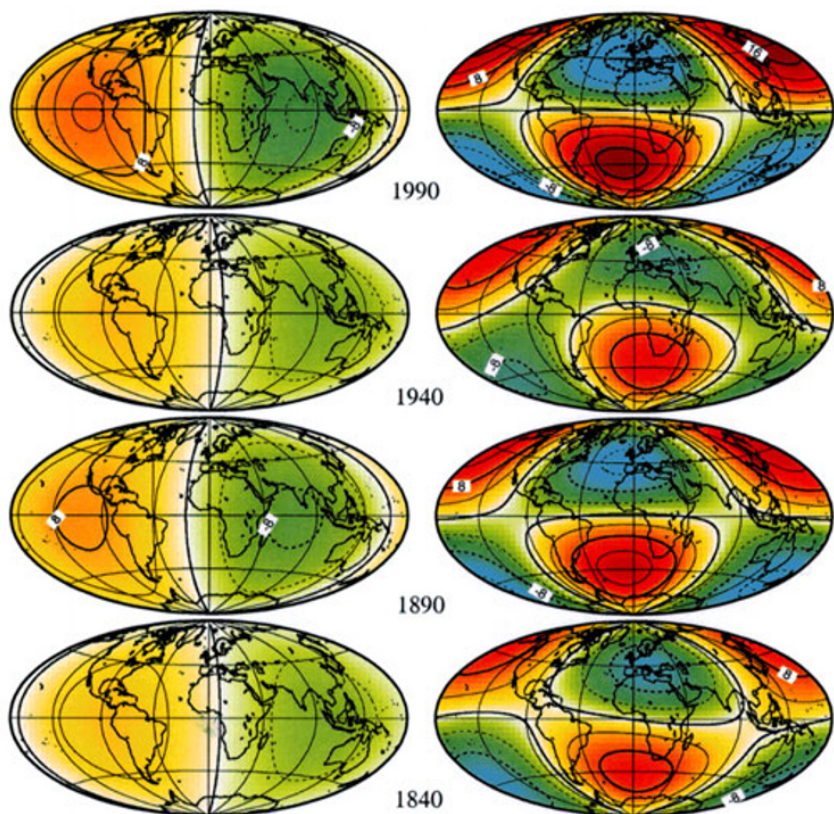

1890
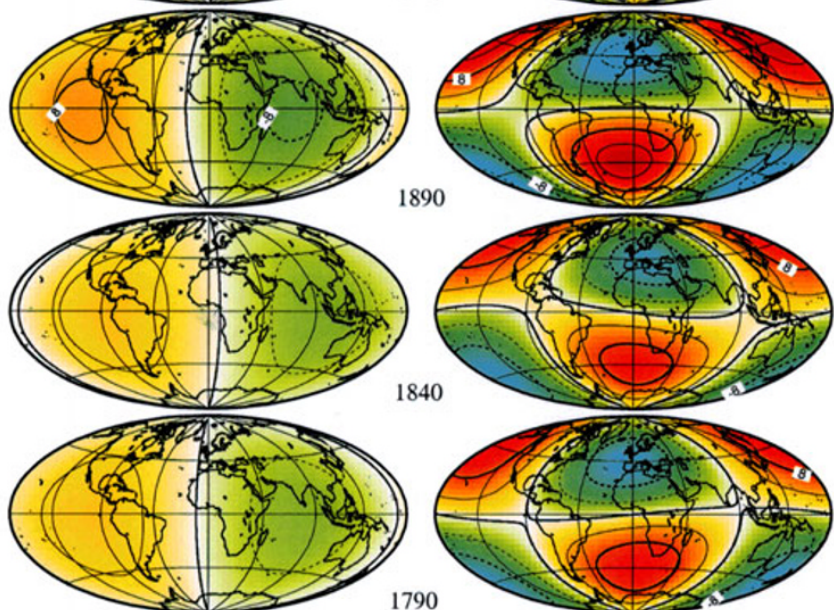

1840
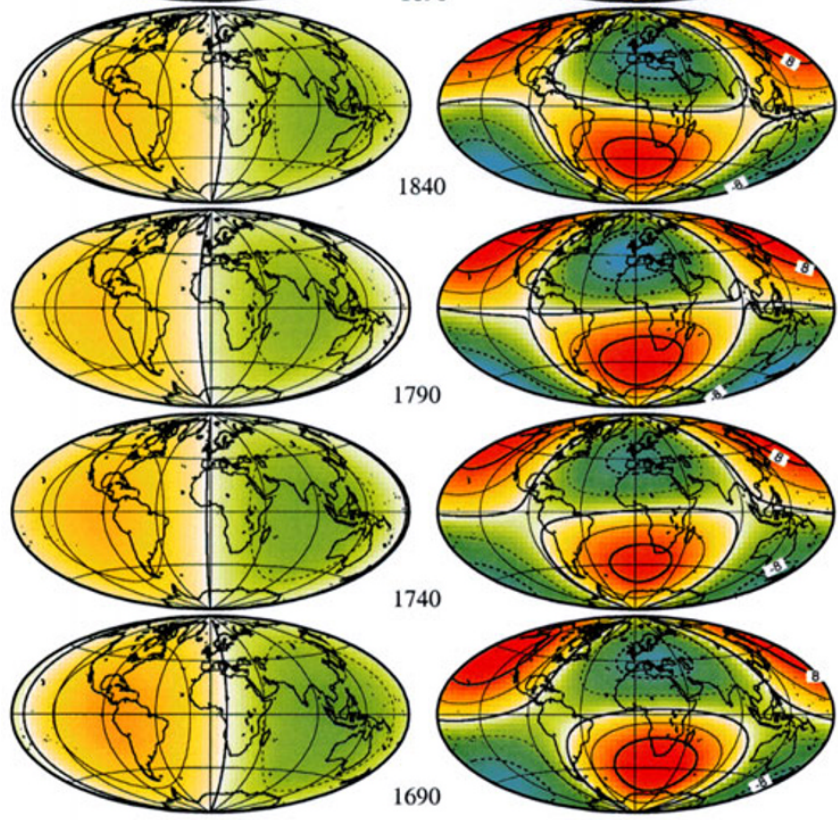

Fig. 3. Degree 1 of the poloidal (left) and degree 2 of the nonzonal toroidal (right) components of the flow at the CMB. Contour interval: $4 \cdot 10^{-4}$ rad $y r^{-1}$. 



Fig. 4. Auto-correlation coefficients for different degrees of the poloidal (left) and toroidal (right) components of the flow at the CMB: $n=1$ (solid line), $n=2$ (dotted line), $n=3$ (dashed line) and $n=4$ (dot-dashed line).

harmonics; the zonal toroidal terms will be considered separately for reasons given below). Clearly, the geometry of these components has remained remarkably constant over the period under study (in the case of $Y_{1}^{1}$, only the stability of the origin meridian is of course significant).

As in the case of the field itself, we have computed the correlation function for each degree of the motion. The function $K_{n}^{s}$ is computed for the poloidal part by Eq. (1) where $g_{n}^{m}$, $h_{n}^{m}$ are replaced by $s_{n}^{m c}$, and $s_{n}^{m s}$; the function $K_{n}^{t}$ is computed for the toroidal part also by Eq. (1) where $g_{n}^{m}, h_{n}^{m}$ are replaced by $t_{n}^{m c}$, and $t_{n}^{m s}$. Figure 4 shows the results. Clearly the correlation coefficients $K_{1}^{s}(\tau)$ and $K_{2}^{t}(\tau)$ have a behavior different from the others and remain close to 1 for the whole time-span. This means in particular that the geostrophic elementary vector:

$$
a \vec{V}_{1}^{1}=a\left(\vec{S}_{1}^{1}+\eta \vec{T}_{2}^{1}\right)
$$

( $\eta$ is a known coefficient; for its expression see Le Mouël et al., 1985; Gire and Le Mouël, 1990), is a stable component of the expansion (10), within a varying amplitude $a$.

We have also computed the mean flow over the 1690 1990 time span. Figure 5 shows the average poloidal and toroidal scalars. Clearly $\vec{S}_{1}^{1}$ and $\vec{T}_{2}^{1}$ are heavily present in the average flow; and the flow has a large ingredient symmetrical with respect to the equator. Figures 6 and 7 represent respectively the equatorially symmetric and equatorially anti-symmetric parts of the poloidal and toroidal scalars (see Hulot et al., 1990) for conventions about the symmetric and anti-symmetric parts of the flow). The symmetric part of $\vec{u}$ is quite close to $\vec{V}_{1}^{1}$ (in fact $\vec{V}_{1}^{1 s}$; the symmetry with respect to the Greenwich meridian could appear strange; it is not more

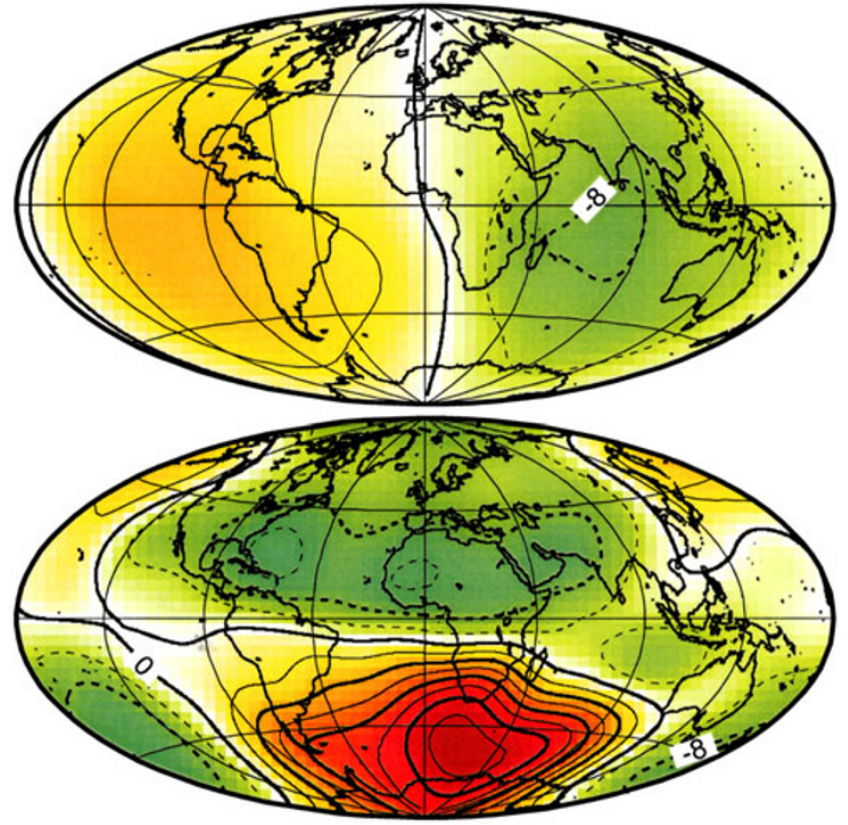

Fig. 5. Poloidal (top) and toroidal (bottom) scalars of the motion averaged over three centuries (1690-1990). Contour interval: $4 \cdot 10^{-4} \mathrm{rad}_{\mathrm{yr}} \mathrm{r}^{-1}$.



Fig. 6. Equatorially symmetrical part of the poloidal (top) and toroidal (bottom) scalars of the average motion over three centuries (1690-1990). Contour interval: $4 \cdot 10^{-4} \mathrm{rad} y r^{-1}$.

than for any other meridian). Nevertheless the antisymmetric component is not negligible (the energy associated with this component represents about $30 \%$ of the energy of the total average flow) and contains essentially $Y_{2}^{1}$ and $Y_{3}^{2}$ for the poloidal scalar and $Y_{1}^{1}, Y_{3}^{1}, Y_{2}^{2}, Y_{4}^{2}$ for the toroidal one. In other words, the antisymmetric part of the mean flow consists essentially of the sum of two elementary geostrophic vectors: 



Fig. 7. Equatorially anti-symmetrical part of the poloidal (top) and toroidal (bottom) scalars of the motion averaged over three centuries (1690-1990). Contour interval: $4 \cdot 10^{-4} \mathrm{rad}_{\mathrm{yr}} \mathrm{r}^{-1}$.

$$
\begin{aligned}
b \vec{V}_{2}^{1}+d \vec{V}_{3}^{2}= & b\left(\vec{S}_{2}^{1}+\zeta^{\prime} \vec{T}_{1}^{1}+\eta^{\prime} \vec{T}_{3}^{1}\right) \\
& +d\left(\vec{S}_{3}^{2}+\zeta^{\prime \prime} \vec{T}_{2}^{2}+\eta^{\prime \prime} \vec{T}_{4}^{2}\right)
\end{aligned}
$$

Figure 8 represents the poloidal and the toroidal scalars corresponding to $a \vec{V}_{1}^{1}+b \vec{V}_{2}^{1}+d \vec{V}_{3}^{2}$ (to be compared with Fig. 5).

To summarize this section, let us say that the flow at the surface of the CMB, taken on average over a few hundreds of years, consists basically of the weighted sum of three elementary geostrophic vectors, one symmetric, $\vec{V}_{1}^{1}$, and the two other ones antisymmetric, $\vec{V}_{2}^{1}$ and $\vec{V}_{3}^{2}$, with respect to the equator. Looking again at Fig. 4, it would be tempting to conjecture that $\vec{V}_{1}^{1}$ has a longer life time and that $\vec{V}_{1}^{1}$ alone gives, in the main, the average geometry of the flow on larger time spans (larger than a few $10^{2}$ years).

Remark The models of the fluid flow at the core surface depend obviously on the quality of the geomagnetic field and secular variation models they are computed from. When using other geomagnetic field and secular variation models (those of Braginsky, 1972; Benkova et al., 1974; and Barraclough, 1974), we find that the morphologies of the corresponding fluid flows, at a given epoch, are quite similar and that the above mentioned characteristics are the same. In fact, the main features of the fluid flow at the CMB can be obtained from secular variation data provided by a distribution of stations as heterogeneous as that of the present-day observatories. For example, using 1959-1986 annual means from 74 observatories, we constructed a secular variation model for every year of the 1960-1985 period in the form of a spherical harmonic expansion up to degree 5 (and degree 1 for the external signal) and derived a fluid flow whose morphology and energy evolution are the same as for the flow

Fig. 8. Poloidal (top) and toroidal (bottom) scalars of the motion corresponding to the sum of three geostrophic vectors, $a \vec{V}_{1}^{1}, b \vec{V}_{2}^{1}$ and $d \vec{V}_{3}^{2}$. Contour interval: $4 \cdot 10^{-4} \mathrm{rad} \mathrm{r}^{-1}$.

model derived from the BJ field models (with a much more homogeneous distribution of the observations). This remark underlines the rapid convergence of computations made in the frozen-flux and tangentially geostrophic approximations. In fact, the main features of the flow depend on a small number of parameters (six for the average flow $a, b, d$ and vector phases; only three if one fixes the symmetry with respect to $\phi=0)$.

\section{The Geomagnetic Jerks and the Behavior of the Flow during the Last Few Decades}

We have considered up to now the evolution of the flow over the whole period where direct observations are available. We will now focus on the last few decades, for which a much finer time sampling is possible. Firstly, we will summarize the methods used to study the so-called geomagnetic jerks. Secondly, we will compare the geometry of successive jerks and the geometry of the velocity flow and the acceleration field, in order to study the relationship between the velocity flow prevailing between the geomagnetic jerks and the acceleration field at the times of these jerks. Finally, we will investigate the space-time variation of the flow at the coremantle boundary and the consequences on the core-mantle coupling and the length-of-day.

\subsection{The geomagnetic jerks}

Examination of geomagnetic data from worldwide observatories has revealed sudden changes in the trend of the secular variation which have been called "geomagnetic jerks" or "secular variation impulses" and have been discussed by a number of authors (Courtillot et al., 1978; Malin and Hodder, 1982; Malin et al., 1983; Courtillot and Le Mouël, 1984; Kerridge and Barraclough, 1985; McLeod, 1985; Gavoret et al., 1986; Gubbins and Tomlinson, 1986; Whaler, 1987; Golovkov et al., 1989; Stewart and Whaler, 1992). These 




Fig. 9. Thick solid line: Synthetic signal composed of 3 jerks located at $t_{0}=(500,1000,1500)$ with regularities $\alpha=(1.4,1.6,1.5)$ and amplitudes $\beta=(+1.0,-0.75,+1.5)$. Three lines are extrapolations of the signal obtained by extinguishing successively jerks: the thin solid line (a) is what the signal would have been in the absence of jerks 1, 2, and 3; the dotted line (b) is what the signal would have been if jerks 2 and 3 were absent, and the dashed line (c) is what the signal would have been if jerk 3 was absent.

former analyses established the global character and the internal origin of the jerks. In order to recover more accurate information about these events (time of occurrence, duration, distribution, and other characteristics), we recently applied a wavelet analysis to geomagnetic time series from about one hundred observatories (Alexandrescu et al., 1995, 1996). We found that seven such events took place during the 1900-1990 period, two of which at least (1969 and 1979) could be described as global in character. A more recent geomagnetic jerk occurring in 1992 has been pointed out by Macmillan (1996) and De Michelis et al. (1998), which has also a worldwide character (Le Huy et al., 1998).

All the events, everywhere, for each component of the geomagnetic field, reveal a singular behavior with a regularity close to 1.5 . Let us recall what this means. A general expression for the jerk signal is:

$$
j\left(t \mid \alpha, \beta, t_{0}\right)=\beta H\left(t-t_{0}\right)\left(t-t_{0}\right)^{\alpha},
$$

where $H(t)$ is the Heaviside distribution, $\beta$ is an amplitude factor, $t_{0}$ is the starting date of the jerk, and $\alpha$ is its regularity. The larger the regularity, the smoother the variation of the function. Let us, for illustration, consider a signal $f(t)$ made of 3 jerks:

$$
\begin{aligned}
f(t)= & j(t \mid 1.4,1,500)+j(t \mid 1.6,-0.75,1000) \\
& +j(t \mid 1.5,1.5,1500) .
\end{aligned}
$$

This signal is shown on Fig. 9. Using wavelet transform allows us to retrieve both the times of the jerks and the regularities $\alpha(1.4,1.6,1.5$ in the example); it also gives the amplitudes $\beta(1.0,-0.75,1.5)$ in this synthetic case. In the case of real data a long series of homogeneous monthly mean values is required to provide good estimates of $\beta$. Too few series of this kind exist to allow maps of the jerk amplitude (for the three components) to be safely drawn.

So we have used a cruder technique in order to take advantage of as many observatories as possible. Let us then recall the technique used by Le Huy et al. (1998). It is considered that in a given time interval $\left[t_{1}, t_{2}\right]$, only one geomagnetic jerk exists at time $t_{0}$. For a given $t_{0}$, for each observatory and for each component $(\dot{X}, \dot{Y}, \dot{Z})$, two straight-line segments were computed which best fit the data in the least-squares sense for the time intervals before and after $t_{0}$ :

$$
\begin{array}{cc}
\dot{E}(t)=a_{1} t+b_{1} & t \leq t_{0} \\
\dot{E}(t)=a_{2} t+b_{2} & t \geq t_{0} .
\end{array}
$$

The amplitude of the geomagnetic jerk $\delta \ddot{E}$, for each component, at each observatory, is defined as the difference between the coefficients $a_{2}$ and $a_{1}$. The obtained values are of the order of a few $n T y r^{-2}$. The three components $\delta \ddot{X}, \delta \ddot{Y}$ and $\delta \ddot{Z}$, for the three jerks, are then submitted to a spherical harmonic analysis up to degree 4 (see Le Huy et al., 1998 for details). We checked that the wavelet analysis and Le Huy et al. techniques provide results in reasonable agreement.

Taking the time derivative of Eq. (2) just before and just after the occurrence time of a jerk, $t_{0}$, and assuming that $B_{r}$, $\dot{B}_{r}(\alpha>1)$ and $\vec{u}$ are continuous at $t=t_{0}$ :

$$
\ddot{B}_{r}^{+}-\ddot{B}_{r}^{-}=-\vec{\nabla}_{H} \cdot\left[\left(\overrightarrow{\dot{u}}^{+}-\overrightarrow{\dot{u}}^{-}\right) B_{r}\right]
$$

i.e.:

$$
\delta \ddot{B}_{r}=-\vec{\nabla}_{H} \cdot\left(\delta \vec{\gamma} B_{r}\right)
$$



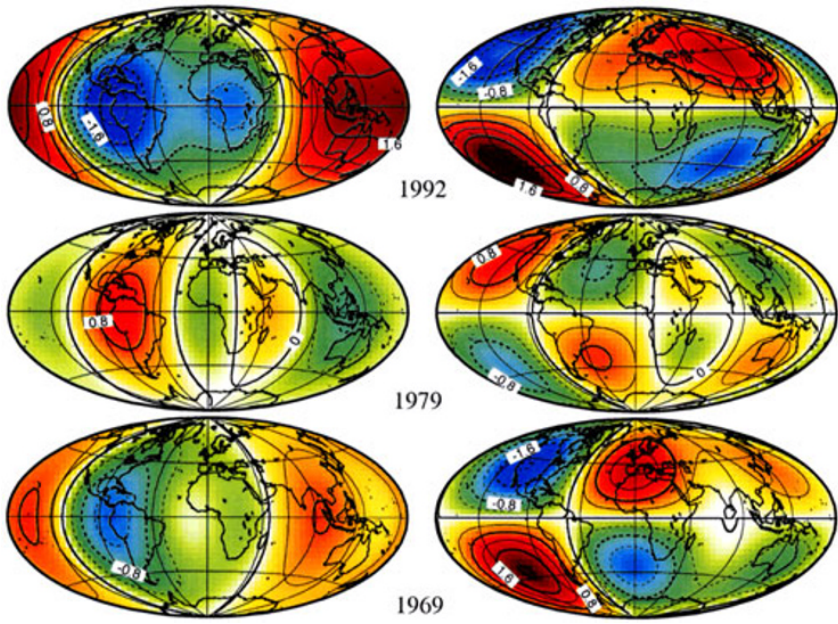

Fig. 10. Equatorially symmetrical part of the poloidal (left) and toroidal (right) scalars of the acceleration jumps corresponding to the three jerks (1969, 1979, 1992). Contour interval: $0.4 \cdot 10^{-4} \mathrm{rad}_{\mathrm{yr}}{ }^{-2}$.
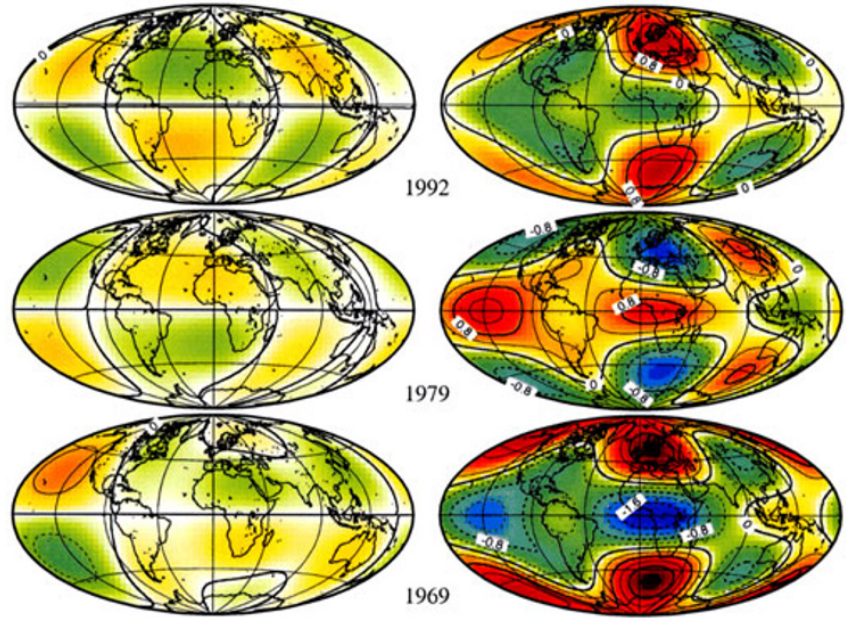

Fig. 11. Equatorially anti-symmetrical part of the poloidal (left) and toroidal (right) scalars of the acceleration jumps corresponding to the three jerks (1969, 1979, 1992). Contour interval: $0.4 \cdot 10^{-4} \mathrm{rad}_{\mathrm{r}} \mathrm{r}^{-2}$.
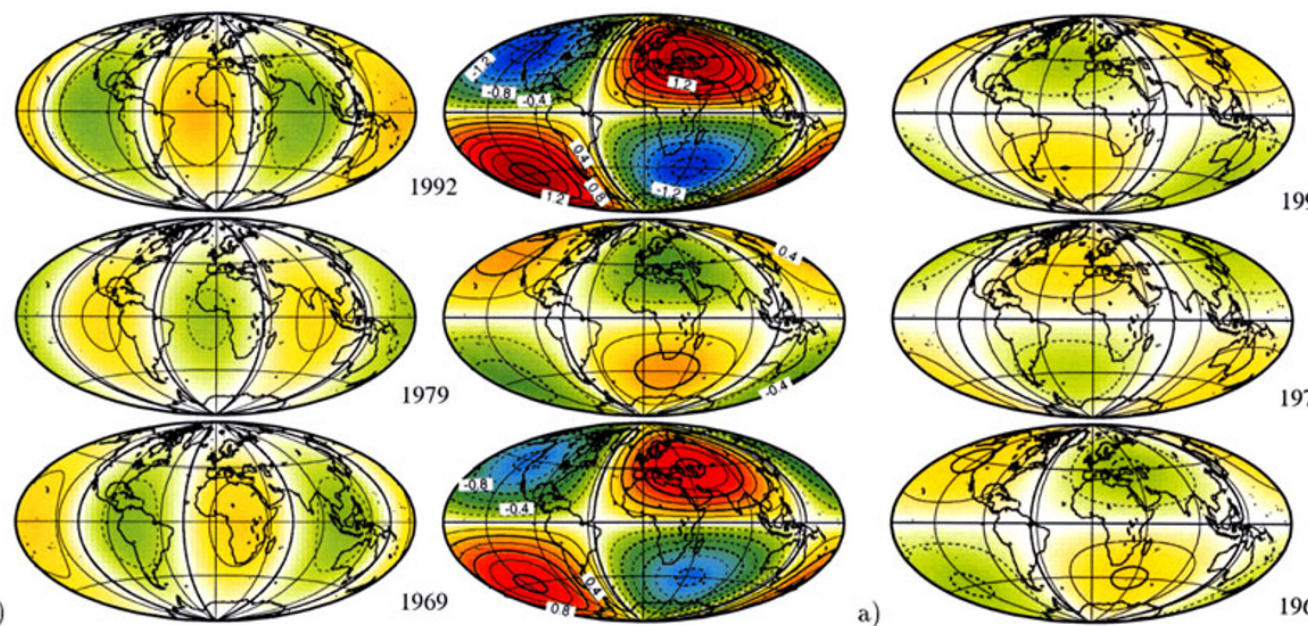

1992
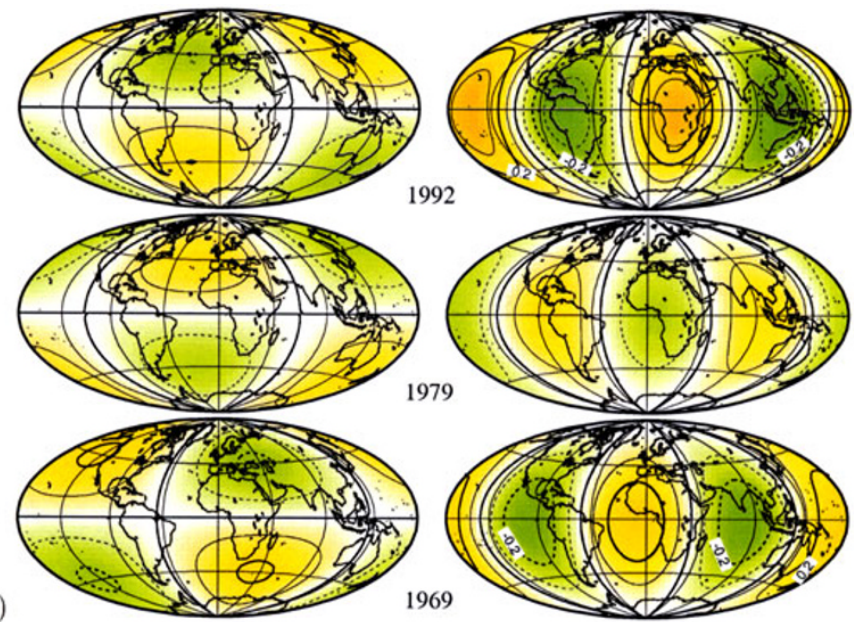

1979
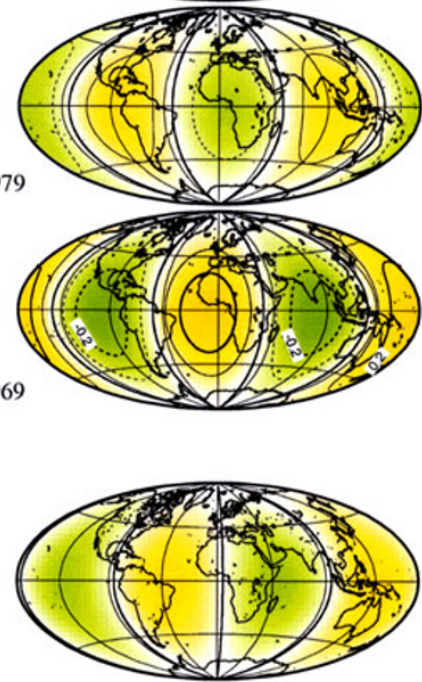

b)


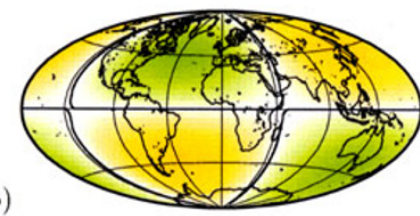

b)

Fig. 13. a) Degree 2 component of the equatorially anti-symmetric part of the poloidal (left) and toroidal (right) scalars of acceleration jumps corresponding to the three jerks $(1969,1979,1992)$. Contour interval: $0.1 \cdot 10^{-4} \mathrm{rad} \mathrm{r}^{-2}$. b) Degree 2 component of the equatorially anti-symmetric part of the poloidal (left) and toroidal (right) scalars of the 1990 flow. Contour interval: $2 \cdot 10^{-4} \mathrm{rad} y r^{-1}$. where the superscripts ".-" and "+" refer to the values before and after the jerk, $\delta \ddot{B}_{r}$ is the radial component of the geomagnetic jerk at the $\mathrm{CMB}$, and $\delta \vec{\gamma}$ is the corresponding jump in the acceleration of the fluid flow at the CMB. The model of $B_{r}$ is again that of Bloxham and Jackson (1992) for the three epochs: 1969, 1979 and 1992 (the model for the latter date is extrapolated from the models of the main field and secular variation for 1990). $\delta \ddot{B}_{r}$ is given by the model of $\vec{\delta} \ddot{B}$ computed as described above from $(\delta \ddot{X}, \delta \ddot{Y}$ and $\delta \ddot{Z}) . B_{r}$ and $\delta \ddot{B}_{r}$ being known, and the tangential geostrophic hypothesis allowing us to again use equation (4) with $\delta \vec{\gamma}$ in place of $\vec{u}$, equations (17) and (4) allow us to compute $\delta \vec{\gamma}$. The jump $\delta \vec{\gamma}$ can, as in the case of $\vec{u}$, be expressed as the sum of poloidal and toroidal ingredients (see Eq. (5) where $\vec{u}$ becomes $\delta \vec{\gamma}$ ), and expanded into spherical harmonics (Eq. (6) with $\delta \vec{\gamma}$ instead of $\vec{u}$ ). $\delta \vec{\gamma}$ can also be expanded on the basis 

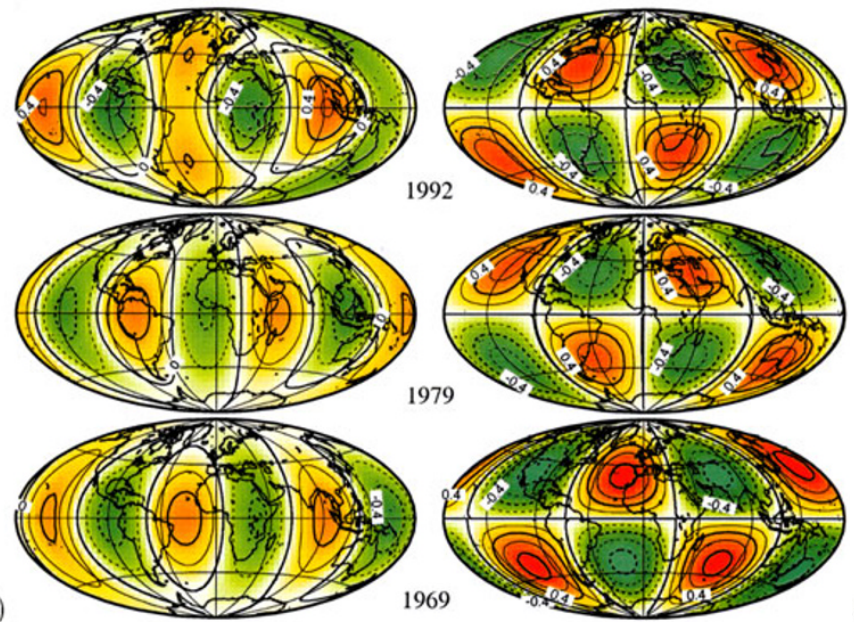

992


a)


1992
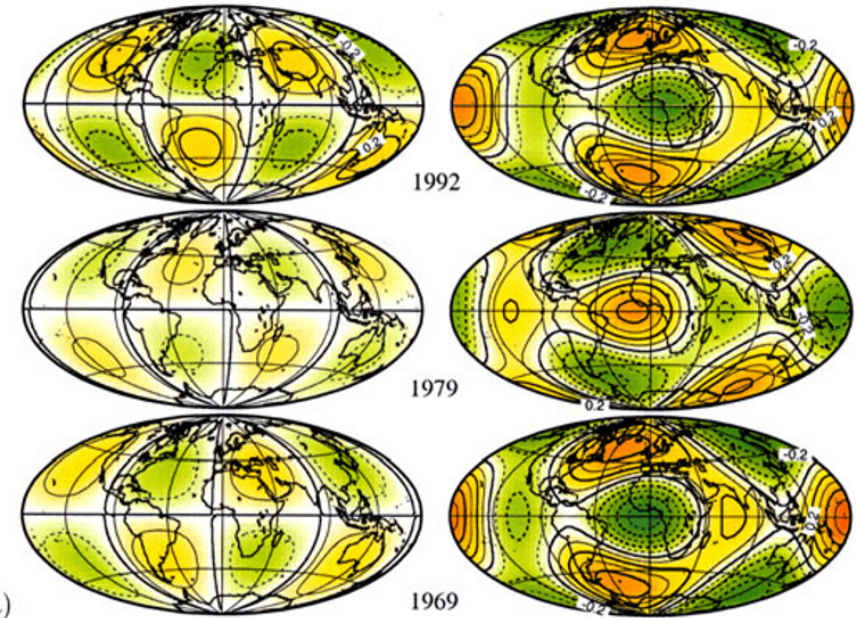

1969



b)
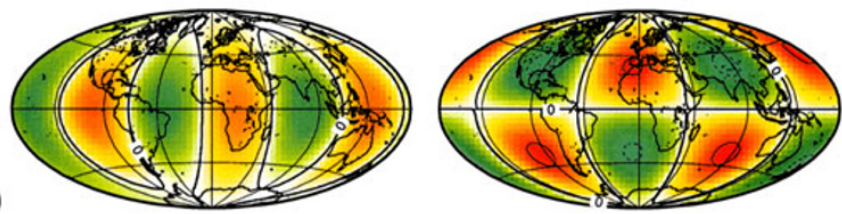

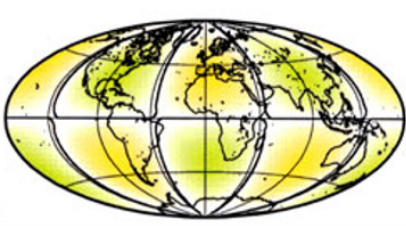

b)
Fig. 14. a) Degree 3 component of the equatorially symmetric part of the poloidal (left) and toroidal (right) scalars of acceleration jumps corresponding to the three jerks $(1969,1979,1992)$. Contour interval: $0.2 \cdot 10^{-4} \mathrm{rad} y r^{-2}$. b) Degree 3 component of the equatorially symmetric part of the poloidal (left) and toroidal (right) scalars of the 1990 flow. Contour interval: $4 \cdot 10^{-4} \mathrm{rad} \mathrm{yr}^{-1}$.

of elementary geostrophic vectors as in Eq. (10).

\subsection{Comparison of the geometry of successive jerks}

We will focus on the 1969, 1979, 1992 jerks since they are by far the best documented. We have already discussed this comparison in Le Huy et al. (1998); we now elaborate on this and emphasize outstanding features.

Figure 10 shows the equatorially symmetric parts of the poloidal and toroidal scalars corresponding to the three jerks. The geometry is broadly the same for the three jerks, with a change of sign in 1979 compared to 1969 and 1992 . Figure 11 is the same for the equatorially anti-symmetric part. The similarity of the geometries, within the sign change in 1979, is even more striking, especially for the toroidal scalar. Figures 12 (a), 13 (a), 14 (a), 15 (a) show the same pictures separately for the components of degrees 2 and 3 of the poloidal and toroidal scalars, for the three jerks. The similarity of the 1969, 1979 and 1992 figures, within a sign change in 1979, is conspicuous (despite some observable phase shifts).

\subsection{Comparison of the geometries of the velocity flow and the acceleration field}

Let us now compare the geometry of the flow $\vec{u}$ determined at a given time - we chose 1990, the last date of the BJ model - with the geometry of the jerks (the same for all three in a first gross approximation - as described above). For this comparison we retain only the low degree terms (the expansion of $\vec{u}$ goes to degree 13 , while the expansion of $\delta \vec{\gamma}$ goes only up to degree $4 ; \delta \ddot{B}_{r}$ models, obtained as described above, are more difficult to get and less accurate than models of secular variation). Figures 12 (b), 13 (b), 14 (b), 15 (b) show the degree 2 and 3 components of the equatori-
Fig. 15. a) Degree 3 component of the equatorially anti-symmetric part of the poloidal (left) and toroidal (right) scalars of acceleration jumps corresponding to the three jerks $(1969,1979,1992)$. Contour interval: $0.1 \cdot 10^{-4} \mathrm{rad}_{r^{-2}}$. b) Degree 3 component of the equatorially anti-symmetric part of the poloidal (left) and toroidal (right) scalars of the 1990 flow. Contour interval: $2 \cdot 10^{-4} \mathrm{rad} \mathrm{r}^{-1}$.

ally symmetric part and the equatorially anti-symmetric part of the poloidal scalar of the 1990 flow $\vec{u}$, to be compared with the same figures (a).

The components of the flow and of the acceleration jump for the same degrees have similar morphologies. However, there is a distinct shift in longitude (about $40-50^{\circ}$ ), between the flow and the acceleration jump, for a given degree.

\subsection{Space time variation of the flow at the CMB for the} last three decades

1) In previous papers (Alexandrescu et al., 1995, 1996) it was shown that the regularity of the jerks is the same $(\approx$ $3 / 2$ ) for the three components of the field, everywhere at the Earth's surface, both for the 1969 and 1979 events and it seems to be again the same in 1992 (in most observatories the lengths of the geomagnetic series after this date are not long enough to allow a proper analysis of this last jerk by wavelets). It results that each jerk component of $\vec{B}(\theta, \phi, t)$ ( $\left.B_{x}, B_{y}, B_{z}\right)$ defined as in Eq. (13) is of the form:

$$
B_{i j}(\theta, \phi, t)=b_{i j}(\theta, \phi) H\left(t-t_{j}\right)\left(t-t_{j}\right)^{3 / 2}
$$

where $j=1969,1979,1992, i=x, y, z$.

2) The present study shows that the acceleration jump jerk - is, in a first approximation, the same for the three events $t_{j}$ within a sign change. From the remarks 1) and 2) and the form of the induction equation, it results that a simple schematic representation of the velocity field $\vec{u}$ at the CMB, from a few years before 1969 up to now is:

$$
\begin{aligned}
\vec{u}(t, \theta, \phi)= & \vec{u}_{0}(t, \theta, \phi) \\
& +\left[\eta_{1} H\left(t-t_{1}\right) \cdot\left(t-t_{1}\right)^{1 / 2}\right.
\end{aligned}
$$






Fig. 16. Behavior of length-of-day monthly means averaged over five years (dashed line) and of residuals of a quadratic regression applied to a synthetic signal composed of 3 jerks (see also Fig. 9) located at $t_{0}=(1969,1979,1992)$ with the regularities $\alpha=(1.5,1.5,1.5)$ and amplitudes $\beta=(+1.0,-1.0,+1.0)$ (solid line).

$$
\begin{aligned}
& +\eta_{2} H\left(t-t_{2}\right) \cdot\left(t-t_{2}\right)^{1 / 2} \\
& \left.+\eta_{3} H\left(t-t_{3}\right) \cdot\left(t-t_{3}\right)^{1 / 2}\right] \delta \vec{\gamma}(\theta, \phi)(19)
\end{aligned}
$$

where the coefficients $\eta_{1}, \eta_{2}, \eta_{3}$, with dimension $L T^{-3 / 2}$ are the amplitudes of the $t_{1}, t_{2}, t_{3}$ jerks in the velocity field. $\delta \vec{\gamma}$ (dimensionless) characterises the geometry of the jerk in the velocity $\vec{u}$ (regularity $1 / 2$ ).

\subsection{Consequences on the core-mantle coupling and length-of-day}

From Eq. (3), the pressure $p$ has a similar expression to (19):

$$
\begin{aligned}
p(t, \theta, \phi)= & p_{0}(t, \theta, \phi)+\left[\eta_{1} H\left(t-t_{1}\right) \cdot\left(t-t_{1}\right)^{1 / 2}\right. \\
& +\eta_{2} H\left(t-t_{2}\right) \cdot\left(t-t_{2}\right)^{1 / 2} \\
& \left.+\eta_{3} H\left(t-t_{3}\right) \cdot\left(t-t_{3}\right)^{1 / 2}\right] \\
& \cdot \delta p(\theta, \phi) \rho \Omega_{0} c
\end{aligned}
$$

where $\rho$ is density, $\Omega_{0}$ the Earth's mean rotation and $\delta p(\theta, \phi)$ (dimensionless) characterizes the geometry of the jerk on pressure (regularity $1 / 2$ ).

Let us suppose that the axial torque exerted on the mantle by the core is essentially a topographic torque (Hide, 1989; Jault and Le Mouël, 1989, 1999; Hide et al., 1993):

$$
\Gamma(t)=-\iint_{C M B} p(t, \theta, \phi) \frac{\partial h}{\partial \phi}(\theta, \phi) \mathrm{d} S
$$

where $h$ is the deviation of the CMB from the mean sphere.

Whence, due to the fact that $h(\theta, \phi)$ is constant in time, it comes:

$$
\begin{aligned}
\Gamma(t)= & \Gamma_{0}(t)+\left[\eta_{1} H\left(t-t_{1}\right) \cdot\left(t-t_{1}\right)^{1 / 2}+\eta_{2} H\left(t-t_{2}\right)\right. \\
& \left.\cdot\left(t-t_{2}\right)^{1 / 2}+\eta_{3} H\left(t-t_{3}\right) \cdot\left(t-t_{3}\right)^{1 / 2}\right] \Gamma_{1}
\end{aligned}
$$

with

$$
\begin{gathered}
\Gamma_{0}(t)=-\iint_{C M B} p_{0}(t, \theta, \phi) \frac{\partial h}{\partial * \phi}(\theta, \phi) \mathrm{d} S \\
\Gamma_{1}=-\rho \Omega_{0} c \iint_{C M B} \delta p(\theta, \phi) \frac{\partial h}{\partial \phi}(\theta, \phi) \mathrm{d} S .
\end{gathered}
$$

Now, from $I_{m} d \Omega / d t=\Gamma$, it comes:

$$
\begin{aligned}
\Omega(t)= & \Omega\left(t_{0}\right)+\int_{t_{0}}^{t}\left(\Gamma_{0}(t) / I_{m}\right) d t+\frac{2}{3} \frac{\Gamma_{1}}{I_{m}} \\
& {\left[\eta_{1} H\left(t-t_{1}\right)\left(t-t_{1}\right)^{3 / 2}+\eta_{2} H\left(t-t_{2}\right)\left(t-t_{2}\right)^{3 / 2}\right.} \\
& \left.+\eta_{3} H\left(t-t_{3}\right)\left(t-t_{3}\right)^{3 / 2}\right] .
\end{aligned}
$$

The term $\int_{t_{0}}^{t}\left(\Gamma_{0}(t) / I_{m}\right) d t$ is actually unknown. It includes not only the effect of a smoothly changing pressure on a standing topography $h(\theta, \phi)$, but also the effect of the displacement - due to rotation itself — of the pressure field $p_{0}$ with respect to $h$ (Jault and Le Mouël, 1991). It has to be supposed, if the topography is as large as given by some seismologists (Morelli and Dziewonski, 1987; Forte et al., 1995), that the value of $\Gamma_{0}$ is much smaller than the value which could be derived from order of magnitude computations; some orthogonality relationship between $p_{0}$ and $h$ must be satisfied. Such a relation between $\delta p$ and $h$ is not assumed; there is a quite significant phase shift between the jerk acceleration and the flow (Fig. 12 to 15), and the jerk contribution is smaller (the contribution of the $t_{j}$ jerk is just zero at time $t_{j}$ ).

Therefore we just compute a smooth simple trend to represent $\Omega\left(t_{0}\right)+\int_{t_{0}}^{t}\left(\Gamma_{0}(t) / I_{m}\right) d t$ in such a way that adding to it 
the jerk signal (3rd member of the right hand side of Eq. (25)) gives a good representation of the length-of-day from, say, 1960. The essential point is that the singularities are in the jerk signal; the same regularity $\alpha$ is assumed for each singularity. The results are illustrated in Fig. 16. The 1969, 1979, 1992 magnetic events are followed by maxima in $\Omega$, with a lag of a few years (about 3 years). This is to be compared with the features of the real data curve, as was done by Pais et al., 1999. Note that singularities with regularities $\alpha=3 / 2$ should be found in length-of-day data at time $t_{j}$. The present reasoning remains of course qualitative.

\section{Conclusion}

We have tried to single out first-order gross features of the spatio-temporal behavior of the flow at the CMB computed in the frozen-flux and tangential geostrophic approximations. The geometry as well as the temporal variation look - to this approximation - very simple. The flow can indeed be represented, for its main part, by the sum of a few geostrophic elementary vectors. Coming to a more accurate description of the last three decades, the geometries of the acceleration flows corresponding to the three jerks of 1969, 1979, 1992 are similar, within a sign change for the 1979 event. Similarities also exist between the geometry of the acceleration jumps and the flow itself. The memory of the jerk flows over ten year time intervals is probably not to be attributed to some kind of interaction with the mantle through the CMB. More probably, it is a consequence of the existence of simple standing features of the flow. No physical interpretation has been tempted here. We have also derived an analytical schematic representation of the flow field for the last three decades which could account for some characteristics of the decade of length-of-day variations. As usual, the inadequacy of the data appears as a main limitation to the analysis. Combining simultaneous satellite and observatory data will in the near future help in better understanding these processes.

Acknowledgments. We thank Hisayoshi Shimizu and David Barraclough for their helpful comments on an earlier version of the manuscript. This is IPGP contribution 1642.

\section{References}

Alexandrescu, M., D. Gibert, G. Hulot, J. -L. Le Mouël, and G. Saracco, Detection of geomagnetic jerks using wavelet analysis, J. Geophys. Res., 100(B7), 12557-12572, 1995.

Alexandrescu, M., D. Gibert, G. Hulot, J. -L. Le Mouël, and G. Saracco, Worldwide wavelet analysis of geomagnetic jerks, J. Geophys. Res., 101(B10), 21975-21994, 1996.

Backus, G. E., Poloidal and toroidal fields in geomagnetic field modeling, Rev. Geophys., 24, 75-109, 1986.

Backus, G. E. and J. -L. Le Mouël, The region of the core-mantle boundary where geostrophic velocity fields can be determined from frozen-flux magnetic data, Geophys. J. R. Astron. Soc., 85, 617-628, 1986.

Barraclough, D. R., Spherical harmonic analyses of the geomagnetic field for eight epochs between 1600 and 1910, Geophys. J. R. Astron. Soc., 36, 497-513, 1974.

Benkova, N. P., G. I. Kolomiytseva, and T. N. Cherevko, Analytical model of the geomagnetic field and its secular variation over a period of 400 years (1550-1950), Geomagn. Aeron., 14, 751-755, 1974.

Bloxham, J. and D. Gubbins, The secular variation of Earth's magnetic field, Nature, 317, 777-781, 1985.

Bloxham, J. and A. Jackson, Time-dependent mapping of the magnetic field at the core-mantle boundary, J. Geophys. Res., 97, 19537-19564, 1992.

Braginsky, S. I., Spherical analyses of the main geomagnetic field in 15501800, Geomag. Aeron., 12, 524-529, 1972.
Carlut, J. and V. Courtillot, Geomagnetic paleosecular variation in the last 5 million years: How many multipoles are actually resolvable? (abstract), EOS Suppl., 76 (46), 166 , 1995.

Carlut, J. and V. Courtillot, How complex is the time-averaged geomagnetic field over the past 5 Myr?, Geophys. J. Int, 134, 527-544, 1998.

Chulliat, A. and G. Hulot, Local computation of the geostrophic pressure at the top of the core, Phys. Earth Planet. Inter., 117, 309-328, 2000.

Courtillot, V. and J. -L. Le Mouël, Geomagnetic secular variation impulses, Nature, 311, 709-716, 1984.

Courtillot, V., J. Ducruix, and J. -L. Le Mouël, Sur une accélération récente de la variation séculaire du champ magnétique terrestre, C. R. Acad. Sci. Paris, 287, Série D., 1095-1098, 1978.

De Michelis, P., L. Cafarella, and A. Meloni, Worldwide character of the 1991 jerk, Geophys. Res. Lett., 25, 377-380, 1998.

Forte, A. M., J. X. Mitrovica, and R. L. Woodward, Seismic-geodynamic determination of the origin of excess ellipicity of the core-mantle boundary, Geophys. Res. Lett., 22(9), 1013-1016, 1995.

Fritsche, H., Atlas des Erdmagnetismus, pp. 1-26, Riga, 1903.

Gavoret, J., D. Gibert, M. Menvielle, and J. -L. Le Mouël, Long-term variations of the external and internal components of the Earth's magnetic field, J. Geophys. Res., 91(B5), 4787-4796, 1986.

Gire, C. and J. -L. Le Mouël, Tangentially geostrophic flow at the coremantle boundary compatible with the observed geomagnetic secular variation: the large-scale component of the flow, Phys. Earth Planet. Inter. 59, 259-287, 1990.

Gire, C., J. -L. Le Mouël, and J. Ducruix, Evolution of the geomagnetic secular variation field from the beginning of the century, Nature, 307, 349-352, 1984

Gire, C., J. -L. Le Mouël, and T. Madden, Motions at the core surface derived from secular variation data, Geophys. J. R. Astron. Soc., 84, 1-29, 1986.

Golovkov, V. P., T. I. Zvereva, and A. O. Simonyan, Common features and differences between "jerks" of 1947, 1958 and 1969, Geophys. Astrophys. Fluid Dyn., 49, 81-96, 1989

Gubbins, D. and J. Bloxham, Morphology of the geomagnetic field and implications for the geodynamo, Nature, 325, 509-511, 1987.

Gubbins, D. and L. Tomlinson, Secular variation from monthly means from Apia and Amberley magnetic observatories, Geophys. J. R. Astron. Soc., 86, 603-616, 1986.

Gubbins, D. and P. Kelly, Persistent patterns in the geomagnetic field over the past 2.5 Myr, Nature, 365, 829-832, 1993.

Hide, R., Fluctuations in the Earth's rotation and the topography of the core-mantle interface, Phil. Trans. R. Soc. Lond., A328, 351-363, 1989.

Hide, R., R. W. Clayton, B. H. Hager, M. A. Speith, and C. V. Voorhies, Topographic core-mantle coupling and fluctuations in the Earth's rotation, in Relating Geophysical Structures and Processes: The Jeffreys Volume, edited by K. Aki and R. Dmowska, Geophys. Monog., Amer. Geophys. Un., 76, 107-120, 1993

Hulot, G. and J. -L. Le Mouël, A statistical approach to the Earth's main magnetic field, Phys. Earth Planet. Inter., 82, 167-183, 1994.

Hulot, G., J. -L. Le Mouël, and D. Jault, The flow at the core-mantle boundary: symmetry properties, J. Geomag. Geoelectr., 42, 857-874, 1990.

Hulot, G., J. -L. Le Mouël, and J. Wahr, Taking into account truncation problems and geomagnetic model accuracy in assessing computed flows at the core-mantle boundary, Geophys. J. Int., 108, 224-246, 1992.

Jault, D. and J. -L. Le Mouël, The topographic torque associated with a tangentially geostrophic motion at the core surface and inferences on the flow inside the core, Geophys. Astrophys. Fluid Dyn., 48, 273-296, 1989.

Jault, D. and J. -L. Le Mouël, Core mantle boundary shape: constraints inferred from the pressure torque acting between the core and the mantle, Geophys. J. Int., 101, 233-241, 1990.

Jault, D. and J. -L. Le Mouël, Exchange of angular momentum between the core and the mantle, J. Geomag. Geoelectr., 43, 111-129, 1991.

Jault, D. and J. -L. Le Mouël, Comment on 'On the dynamics of topographical core-mantle coupling' by Weijia Kuang and Jeremy Bloxham, Phys. Earth Planet. Inter., 114, 211-215, 1999.

Kelly, P. and D. Gubbins, The geomagnetic field over the past 5 million years, Geophys. J. Int., 128, 315-330, 1997.

Kerridge, D. J. and D. R. Barraclough, Evidence for geomagnetic jerks from 1931 to 1971, Phys. Earth Planet. Inter, 39, 228-236, 1985.

Le Mouël, J. -L., Outer-core geostrophic flow and secular variation of Earth's geomagnetic field, Nature, 311, 734-735, 1984.

Le Mouël, J. -L., C. Gire, and T. Madden, Motions at the core surface in the geostrophic approximation, Phys. Earth Planet. Inter., 39, 270-287, 1985.

Le Huy, M., M. Alexandrescu, G. Hulot, and J. -L. Le Mouël, On the 
characteristics of successive geomagnetic jerks, Earth Planets Space, 50, 723-732, 1998.

Macmillan, S., A geomagnetic jerk for the early 1990's, Earth Planet. Sci. Lett., 137, 189-192, 1996. Malin, S. R. C., Geomagnetic secular variation and its changes, 1942.5 to 1962.5, Geophys. J. R. Astron. Soc., 17, 415-441, 1969.

Malin, S. R. C. and A. D. Clark, Geomagnetic secular variation, 1962.5 to 1967.5, Geophys. J. R. Astron. Soc., 36, 11-20, 1974.

Malin, S. R. C. and B. M. Hodder, Was the 1970 geomagnetic jerk of internal or external origin?, Nature, 296, 726-728, 1982.

Malin, S. R. C., B. M. Hodder, and D. R. Barraclough, Geomagnetic secular variation: a jerk in 1970, in 75th Anniversary Volume of Ebro Observatory, edited by J. O. Cardús, pp. 239-256, Ebro Obs., Tarragona, Spain, 1983.

McLeod, M. G., On the Geomagnetic jerk of 1969, J. Geophys. Res., 90, 4597-4610, 1985.

Morelli, A. and M. Dziewonski, Topography of the core-mantle boundary and lateral homogeneity of the liquid core, Nature, 325, 678-683, 1987. Pais, A., G. Hulot, M. Mandea Alexandrescu, Does the geomagnetic secular variation anticipate or correlate with decade length of day variations? (Abstract), IUGG Birmingham, 1999.

Roberts, P. H. and S. Scott, On the analysis of the secular variation. 1. A hydromagnetic constraint: theory., J. Geomag. Geoelectr., 17, 137-151, 1965.

Stewart, D. N. and K. Whaler, Geomagnetic disturbance fields: An analysis of observatory monthly means, Geophys. J. Int., 108, 215-223, 1992.

Whaler, K. A., A new method for analysing geomagnetic impulses, Phys. Earth Planet. Inter, 48, 221-240, 1987.

M. Le Huy (e-mail: 1hminh@igp.ncst.ac.vn), M. Mandea (e-mail: mioara@ipgp.jussieu.fr), J.-L.Le Mouël(e-mail: lemouel@ipgp.jussieu.fr), and A. Pais (e-mail: pais@ipgp.jussieu.fr) 